Website: http://revistas.lamolina.edu.pe/index.php/acu/index

(C) Universidad Nacional Agraria La Molina, Lima - Perú

\title{
Elementos extractables con cloruro de potasio y acetato de amonio en suelos ácidos del Perú
}

\author{
Potassium chloride and ammonium acetate extractable elements in acid soils of Perú
}

\author{
Klaus P. Raven Willwater \\ *Autor de correspondencia
}

\begin{abstract}
Resumen
Cantidades de elementos extractables con soluciones de cloruro de potasio $(\mathrm{KCl})$ y acetato de amonio $\left(\mathrm{CH}_{3} \mathrm{COONH}_{4}\right)$ comúnmente se designan como fracción intercambiable del suelo y sirven como índices de disponibilidad de nutrientes para plantas cultivadas y del peligro potencial existente en suelos contaminados. Los objetivos de este estudio fueron evaluar las cantidades de $\mathrm{Al}, \mathrm{Ca}, \mathrm{Cu}, \mathrm{Fe}, \mathrm{H}, \mathrm{Mg}$, Mn y $\mathrm{Zn}$ extractables con $\mathrm{KCl}$ y de $\mathrm{K}$ y Na extractables con $\mathrm{CH}_{3} \mathrm{COONH}_{4}$ en un grupo muy diverso de muestras de suelos ácidos del Perú y describir sus patrones de variación. Las concentraciones extractables promedio decrecieron en el orden $\mathrm{Ca}>\mathrm{Al}>\mathrm{K}>\mathrm{Mg}>\mathrm{Fe}>\mathrm{Na}>\mathrm{H}>\mathrm{Mn}>\mathrm{Zn}>\mathrm{Cu}$, mientras que las fracciones molares extractables medias disminuyeron de acuerdo con la secuencia $\mathrm{Al}>\mathrm{Ca}>\mathrm{K}>\mathrm{Mg}>\mathrm{Na}>\mathrm{H}>\mathrm{Mn}>$ $\mathrm{Fe}>\mathrm{Zn}>\mathrm{Cu}$. Los datos mostraron una variabilidad muy alta. En promedio, las concentraciones $\mathrm{Ca}$ y $\mathrm{Al}$ sumaron $70 \%$ del total. Al añadir K y Mg, se alcanzó $90 \%$. El pH estuvo directamente relacionado con el contenido de Mg y, en orden decreciente, con $\mathrm{Ca}$ y $\mathrm{Na}$. Por otra parte, el pH presentó una relación inversa con los contenidos de $\mathrm{Al}, \mathrm{Cu}, \mathrm{Fe}, \mathrm{H}$ y Zn. Las muestras con $\mathrm{pH}$ menor que tres y mayores contenidos de $\mathrm{Cu}$, $\mathrm{Fe}$ y $\mathrm{Zn}$ del estudio tuvieron una porción soluble en agua significativa del total extractable.
\end{abstract}

Palabras clave: suelo; ácido; extracción; elementos; acidez; cloruro de potasio; acetato de amonio; Perú.

\begin{abstract}
Quantities of potassium chloride $(\mathrm{KCl})$ and ammonium acetate $\left(\mathrm{CH}_{3} \mathrm{COONH}_{4}\right)$ extractable elements are commonly designated as exchangeable in soils and are used as indexes for nutrient availability to cultivated plants and potential hazard in contaminated soils. The objectives of this study were to evaluate the amounts of $\mathrm{KCl}$ extractable $\mathrm{Al}, \mathrm{Ca}, \mathrm{Cu}$, $\mathrm{Fe}, \mathrm{H}, \mathrm{Mg}, \mathrm{Mn}$ and $\mathrm{Zn}$ and $\mathrm{CH}_{3} \mathrm{COONH}_{4}$ extractable $\mathrm{K}$ and $\mathrm{Na}$ in a very diverse group of acid soil samples of Perú and to describe their variation patterns. The average extractable concentrations decreased according to the order $\mathrm{Ca}>\mathrm{Al}>\mathrm{K}$ $>\mathrm{Mg}>\mathrm{Fe}>\mathrm{Na}>\mathrm{H}>\mathrm{Mn}>\mathrm{Zn}>\mathrm{Cu}$, while the mean extractable molar fractions decreased according to the sequence $\mathrm{Al}>\mathrm{Ca}>\mathrm{K}>\mathrm{Mg}>\mathrm{Na}>\mathrm{H}>\mathrm{Mn}>\mathrm{Fe}>\mathrm{Zn}>\mathrm{Cu}$. The data showed a very high variability. On average, the $\mathrm{Al}$ and $\mathrm{Ca}$ concentrations added up to $70 \%$ of the total. When $\mathrm{K}$ and $\mathrm{Mg}$ were included, $90 \%$ was reached. The $\mathrm{pH}$ was directly related to the $\mathrm{Mg}$ content and, in decreasing order, to those of $\mathrm{Ca}$ and $\mathrm{Na}$. On the other hand, the $\mathrm{pH}$ showed an inverse relationship with the $\mathrm{Al}, \mathrm{Cu}, \mathrm{Fe}, \mathrm{H}$, and $\mathrm{Zn}$ contents. The samples with $\mathrm{pH}$ less than three and with the highest $\mathrm{Cu}, \mathrm{Fe}$, and $\mathrm{Zn}$ contents of this study had a significant part of the extractable total in the water soluble form.
\end{abstract}

Keywords: soil; acid; extraction; elements; acidity; potassium chloride; ammonium acetate; Perú.

\section{Introducción}

Soluciones de cloruro de potasio $(\mathrm{KCl})$ y de acetato de amonio $\left(\mathrm{CH}_{3} \mathrm{COONH}_{4}\right)$ han sido utilizadas comúnmente para cuantificar cationes intercambiables en muestras de suelo. Esta información puede servir como índices de disponibilidad de nutrientes para plantas cultivadas y como indicadores de movilidad y biodisponibilidad de metales en suelos contaminados (Alloway, 2013; Mengel y Kirkby, 2001).

Los elementos comúnmente evaluados mediante estas extracciones incluyen $\mathrm{Al}, \mathrm{Ca}, \mathrm{H}, \mathrm{K}, \mathrm{Mg}$ y $\mathrm{Na}$. Han sido publicadas compilaciones mostrando la diversidad de estos resultados para ciertas regiones, países o continentes
(Deressa et al., 2013; Hengl et al., 2015; Lopes y Cox, 1977; Tomasic et al., 2013; etc.). Específicamente, en el Perú, se han generado muchos resultados de este tipo en estudios de suelos y reportes analíticos para agricultores. Sin embargo, no se ha podido encontrar ningún estudio que detalle la diversidad de sus valores en el territorio Peruano. Por otra parte, reportes que incluyan al $\mathrm{Cu}, \mathrm{Fe}, \mathrm{Mn}$ y $\mathrm{Zn}$ son menos comunes a nivel internacional. En el Perú, esta información es aún mas escasa.

Los objetivos del presente trabajo fueron evaluar las concentraciones de $\mathrm{Al}, \mathrm{Ca}, \mathrm{Cu}, \mathrm{Fe}, \mathrm{H}, \mathrm{Mg}, \mathrm{Mn}$ y $\mathrm{Zn}$ extractables con $\mathrm{KCl}$ y de $\mathrm{K}$ y $\mathrm{Na}$ extractables con $\mathrm{CH}_{3} \mathrm{COONH}_{4}$ en un grupo diverso de muestras de suelos 
ácidos del Perú y describir sus patrones de variación. Además de aumentar el conocimiento de las propiedades químicas de los suelos ácidos del Perú, esta información sería útil para la interpretación de resultados de análisis de suelos, específicamente en la elaboración de escalas de calificación de concentraciones de elementos intercambiables en suelos.

\section{Materiales y métodos}

Se colectaron noventa y seis muestras de tierra fina seca al aire de suelos ácidos en diversos lugares del territorio Peruano tratando de maximizar la variabilidad de sus contenidos de materia orgánica, arcilla y solutos y de su $\mathrm{pH}$. La concentración de materia orgánica varió entre 0,1 y $82,2 \%$, con media de $10,6 \%$ y desviación estándar igual a $16,9 \%$. Estos datos fueron obtenidos mediante el método de Walkley-Black, según Bazán (1996). La concentración de arcilla en la tierra fina fluctuó entre 3 y $80 \%$, con promedio igual a $23 \%$ y desviación estándar de $17 \%$. Estos resultados fueron generados mediante el método del hidrómetro, según Bazán (1996). El contenido de solutos fue cuantificado a través de la conductividad eléctrica de suspensiones 1:1 de suelo:agua, en volumen. Este parámetro varió entre 0,02 y $13,68 \mathrm{dS} \mathrm{m} \mathrm{m}^{-1}$, con media de $0,47 \mathrm{dS} \mathrm{m}^{-1}$ y desviación estándar igual a 1,49 $\mathrm{dS} \mathrm{m}^{-1}$. El pH fluctuó entre 2,2 y 6,0 con media igual a 4,5 y desviación estándar de 0,7. El pH fue determinado mediante potenciometría en suspensiones $1: 1$, en masa, de suelo:agua (Thomas, 1996).

La mayor parte de los elementos fue extraída de las muestras de suelo utilizando $\mathrm{KCl} 1 \mathrm{M}$. Las extracciones se realizaron, por duplicado, en las 96 muestras seleccionadas y en 10 blancos sin muestra. Los blancos fueron utilizados para corregir los resultados obtenidos y para calcular los límites de detección y cuantificación en las determinaciones, según lo detallado por Harris (2016).

Cada extracción consistió en equilibrar $15 \mathrm{~g}$ de muestra de suelo con $150 \mathrm{~mL}$ de $\mathrm{KCl} 1 \mathrm{M}$ por 30 minutos. La equilibración se realizó en frascos de polietileno de 250 $\mathrm{mL}$, colocados en posición horizontal en un agitador operado a 190 oscilaciones por minuto. Las suspensiones resultantes fueron filtradas, y el filtrado fue separado en tres porciones. La primera porción de $50 \mathrm{~mL}$ fue destinada a las determinaciones volumétricas de la acidez extractable y de la concentración de $\mathrm{Al}$ mediante el procedimiento de Sims (1996), usando NaF en reemplazo de KF. La segunda porción de $50 \mathrm{~mL}$ fue almacenada en frascos de polietileno de $60 \mathrm{~mL}$ para determinar las concentraciones de $\mathrm{Ca}, \mathrm{Cu}$, $\mathrm{Fe}, \mathrm{Mg}, \mathrm{Mn}$ y $\mathrm{Zn}$. Tres gotas de $\mathrm{HCl}$ concentrado fueron adicionadas a cada frasco con fines de preservación del extracto. La porción remanente de menos de $50 \mathrm{~mL}$ fue almacenada en frascos de polietileno de $60 \mathrm{~mL}$ y utilizada para medir potenciométricamente el $\mathrm{pH}$ y para servir de respaldo.

La concentración de $\mathrm{H}^{+}$extractable ( $\mathrm{HE}, \mathrm{cmol} \mathrm{kg} \mathrm{kg}^{-1}$ ) se calculó a partir del $\mathrm{pH}$ medido en el extracto de $\mathrm{KCl} 1 \mathrm{M}$ para la muestra $(\mathrm{pHm})$ y para el blanco respectivo $(\mathrm{pHb})$ utilizando la siguiente fórmula:

$$
\mathrm{HE}=\frac{1000\left(10^{-\mathrm{pHm}}-10^{-\mathrm{pHb}}\right)}{\gamma_{\mathrm{H}}}
$$

siendo $\gamma_{\mathrm{H}}$ el coeficiente de actividad promedio de los iones $\mathrm{H}^{+}$en el extracto de $\mathrm{KCl}$. El valor usado para $\gamma_{\mathrm{H}}$ fue 1,11 , y se obtuvo de pruebas preliminares con soluciones de $\mathrm{KCl}$ $1 \mathrm{M}$ enriquecidas con $\mathrm{HCl}$. Un valor similar fue reportado por Garrels y Christ (1965) para el $\mathrm{H}^{+}$en soluciones acuosas de fuerza iónica igual a 1. Durante las extracciones de las muestras de suelo, se estima que un máximo de 9,5\% del $\mathrm{K}^{+}$fue consumido para desplazar los cationes nativos del suelo. Por lo tanto, se puede considerar que la concentración de la solución de $\mathrm{KCl}$ se mantuvo cerca de $1 \mathrm{M}$ y actuó como amortiguador de la fuerza iónica de la solución a un valor cercano a 1 .

El K es un elemento abundante en los suelos y dada la imposibilidad de determinar $\mathrm{K}$ en los extractos de $\mathrm{KCl}$ $1 \mathrm{M}$, las 96 muestras de suelo estudiadas también fueron sometidas a una extracción con $\mathrm{CH}_{3} \mathrm{COONH}_{4} 1 \mathrm{M} \mathrm{pH}$ 7. Para ello, se lixiviaron $5 \mathrm{~g}$ de muestra con $100 \mathrm{~mL}$ de solución extractante (Bazán, 1996). En los extractos resultantes, se determinaron las concentraciones de $\mathrm{K}$ y $\mathrm{Na}$

Las concentraciones de $\mathrm{Ca}, \mathrm{Cu}, \mathrm{Fe}, \mathrm{K}, \mathrm{Mg}, \mathrm{Mn}$, Na y Zn en los extractos de $\mathrm{KCl} 1 \mathrm{M} \mathrm{y} \mathrm{CH}_{3} \mathrm{COONH}_{4} 1 \mathrm{MpH} 7$ fueron determinadas mediante espectrofotometría de absorción atómica en llama de acetileno-aire. Las líneas de absorción empleadas fueron 422, 7, 324,8, 248,3, 766,5, 285,2, 279,5, 589,0 y $213,9 \mathrm{~nm}$, respectivamente. Los instrumentos utilizados fueron: 3100 Atomic Absorption Spectrometer (PerkinElmer Inc.), AAnalyst 200 Spectrometer (PerkinElmer Inc.) y contrAA 300 Spectrometer (Analytik Jena $\mathrm{AG}$ ). Para $\mathrm{Ca}$ y $\mathrm{Mg}$, las lecturas fueron realizadas en extractos diluidos conteniendo $1000 \mathrm{mg} \mathrm{L}^{-1}$ de lantano (La). La operación de estos instrumentos se realizó de acuerdo con las recomendaciones de los fabricantes.

Como complemento de las concentraciones de elementos extractables de las muestras de suelo, se evaluó la fracción molar extractable de cada uno de estos. Para ello, se necesitó conocer la suma de concentraciones de elementos extractables. En el presente estudio, esta se definió como la suma de las concentraciones de $\mathrm{Al}, \mathrm{Ca}$, $\mathrm{Cu}, \mathrm{Fe}, \mathrm{H}, \mathrm{Mg}, \mathrm{Mn}$ y $\mathrm{Zn}$ extractables con $\mathrm{KCl} 1 \mathrm{M}$ y de aquellas de $\mathrm{K}$ y $\mathrm{Na}$ extractables con $\mathrm{CH}_{3} \mathrm{COONH}_{4} 1 \mathrm{M} \mathrm{pH}$ 7. Al usar dos soluciones para extraer las mismas sustancias de muestras de suelo, las cantidades obtenidas no deberían ser iguales. Sin embargo, si estas son similares, se podrían combinar con el fin de realizar un análisis aproximado, pero razonable, de los resultados. Estudios realizados por Amacher et al. (1990) y Shuman \& Duncan (1990) sugieren que existe esta similitud.

Los datos de concentraciones de elementos extractables y fracciones molares de estos fueron sometidos a análisis estadísticos descriptivos básicos. Además, se realizaron análisis de correlación lineal simple entre los parámetros evaluados y las variables de selección de suelos utilizadas. 
La significación estadística de los coeficientes de correlación lineal simple (r) se evaluó mediante una prueba de t. Las hipótesis planteada y alternativa en esta fueron $r=$ 0 y $\mathrm{r} \neq 0$, respectivamente. Todos estos análisis estadísticos se realizaron, siguiendo lo descrito por Ott \& Longnecker (2016).

\section{Resultados y discusión}

\section{Estadística descriptiva global}

Los parámetros estadísticos descriptivos para las variables de concentración y de fracción molar de sustancias extractables en la totalidad de las muestras de suelos ácidos estudiadas se presentan en la Tabla 1.

Tabla 1. Estadística descriptiva de concentraciones (cmol $\mathrm{kg}^{-1}$ ) y fracciones molares (mol mol${ }^{-1}$ ) de sustancias extractables en muestras de suelos ácidos del Perú

\begin{tabular}{|c|c|c|c|c|c|}
\hline Sustancia & Mínimo & Máximo & Media & $\begin{array}{c}\text { Desviación } \\
\text { Estándar }\end{array}$ & $\begin{array}{c}\text { Valores No } \\
\text { Detectables } \\
(\%)\end{array}$ \\
\hline \multicolumn{6}{|c|}{ Concentraciones Extractables } \\
\hline $\begin{array}{c}\text { Acidez } \\
\text { Extractable }\end{array}$ & $<0,16$ & 42,0 & 5,78 & 7,27 & 7 \\
\hline $\mathrm{Al}$ & $<0,07$ & 10,9 & 1,48 & 1,75 & 12 \\
\hline $\mathrm{Ca}$ & $<0,01$ & 42,4 & 2,88 & 5,92 & 8 \\
\hline $\mathrm{Cu}$ & $<0,0006$ & 0,657 & 0,00863 & 0,0681 & 77 \\
\hline $\mathrm{Fe}$ & $<0,006$ & 17,5 & 0,201 & 1,79 & 71 \\
\hline $\mathrm{H}$ & 0,00480 & 1,13 & 0,111 & 0,176 & 0 \\
\hline $\mathrm{K}$ & 0,0500 & 4,44 & 0,489 & 0,624 & 0 \\
\hline $\mathrm{Mg}$ & 0,00590 & 3,32 & 0,429 & 0,657 & 0 \\
\hline Mn & $<0,0006$ & 0,565 & 0,0655 & 0,0940 & 1 \\
\hline $\mathrm{Na}$ & 0,0435 & 1,487 & 0,150 & 0,165 & 0 \\
\hline $\mathrm{Zn}$ & $<0,0008$ & 1,85 & 0,0363 & 0,196 & 31 \\
\hline $\begin{array}{c}\text { Suma } \\
\text { Elementos }\end{array}$ & 0,580 & 47,5 & 5,85 & 6,60 & - \\
\hline \multicolumn{6}{|c|}{ Fracciones Molares Extractables } \\
\hline $\mathrm{Al}$ & 0 & 0,941 & 0,421 & 0,309 & - \\
\hline $\mathrm{Ca}$ & 0 & 0,936 & 0,276 & 0,315 & - \\
\hline $\mathrm{Cu}$ & 0 & 0,0596 & 0,000756 & 0,00611 & - \\
\hline $\mathrm{Fe}$ & 0 & 0,775 & 0,0109 & 0,0794 & - \\
\hline $\mathrm{H}$ & 0,000267 & 0,126 & 0,0293 & 0,0243 & - \\
\hline $\mathrm{K}$ & 0,00105 & 0,694 & 0,125 & 0,113 & - \\
\hline $\mathrm{Mg}$ & 0,00394 & 0,383 & 0,0726 & 0,0648 & - \\
\hline $\mathrm{Mn}$ & 0 & 0,111 & 0,0138 & 0,0176 & - \\
\hline $\mathrm{Na}$ & 0,00210 & 0,225 & 0,0463 & 0,0420 & - \\
\hline $\mathrm{Zn}$ & 0 & 0,168 & 0,00442 & 0,0190 & - \\
\hline
\end{tabular}

Las soluciones de $\mathrm{KCl} 1 \mathrm{M}$ y de $\mathrm{CH}_{3} \mathrm{COONH}_{4} 1$ $\mathrm{M} \mathrm{pH} 7$ extraen principalmente formas catiónicas de los elementos evaluados en las muestras de suelo. Las concentraciones extractables incluyen porciones soluble en agua, intercambiable y disuelta de fase sólida. Por lo tanto, los resultados mostrados en la Tabla 1 incluyen estas tres porciones.

Las muestras de suelo y los extractos de $\mathrm{KCl}$ y $\mathrm{CH}_{3} \mathrm{COONH}_{4}$ contienen una mezcla variable de especies para cada elemento. Las formas químicas de estos elementos incluyen especies hidrolíticas mono- a poliméricas y complejos solubles. Estas especies pueden ser de naturaleza catiónica, neutra o aniónica. Por ello, no se puede asignar una carga eléctrica definida a todos los elementos estudiados, y se consideró más apropiado reportar los resultados en términos molares.

La acidez extractable de las muestras de suelos del Perú utilizadas en este estudio tuvo una media igual a 5,78 cmol $\mathrm{H}^{+} \mathrm{kg}^{-1}$ y un coeficiente de variabilidad muy elevado de $126 \%$. Los valores respectivos reportados para otras partes del mundo se resumen en la Tabla 2. En general, la mayoría de los resultados obtenidos en este estudio se encontraron dentro del rango de valores de la bibliografía consultada. Sin embargo, alrededor de un $10 \%$ de los mismos superó al máximo de este rango. El método usado para obtener los valores reportados fue el mismo que se usó en este estudio. Por ello, datos tan extremos aún no han sido reportados en la literatura o algún reporte con este tipo de resultados no ha podido encontrarse en la literatura revisada.

En la Tabla 2, también se resumen concentraciones de elementos intercambiables en muestras de suelos ácidos del mundo. Cabe notar, que estos valores fueron obtenidos usando diversos extractantes, incluyendo soluciones de $\mathrm{KCl}, \mathrm{CH}_{3} \mathrm{COONH}_{4}, \mathrm{BaCl}_{2}, \mathrm{NH}_{4} \mathrm{Cl}, \mathrm{NH}_{4} \mathrm{NO}_{3}$ y $\mathrm{Mg}\left(\mathrm{NO}_{3}\right)_{2}$. Estas soluciones extractantes no llevan a los mismos resultados para una muestra dada. Sin embargo, se ha informado que producen resultados bastante similares (Amacher et al., 1990; Shuman y Duncan, 1990). Aunque estos extractantes solubilizan las tres porciones indicadas previamente, los resultados comúnmente han sido designados como fracciones intercambiables.

Las concentraciones promedio de elementos extractables en muestras de suelos ácidos del Perú disminuyeron en el orden: $\mathrm{Ca}>\mathrm{Al}>\mathrm{K}>\mathrm{Mg}>\mathrm{Fe}>\mathrm{Na}>\mathrm{H}>\mathrm{Mn}>\mathrm{Zn}>$ $\mathrm{Cu}$. Estas medias estuvieron dentro del rango de valores reportados para suelos ácidos de otras partes del mundo. Los valores máximos encontrados para $\mathrm{Al}, \mathrm{H}, \mathrm{Mg}$ y $\mathrm{Na}$ en este estudio también se localizaron dentro del rango de valores reportados. Menos del $6 \%$ de los valores de $\mathrm{Ca}, \mathrm{Cu}$, Fe, K, Mn y Zn lograron superar el respectivo máximo de estos rangos. Por otra parte, los coeficientes de variabilidad para los resultados obtenidos fueron muy altos, superando $100 \%$ en todos los elementos y alcanzando $891 \%$ para el Fe. Esta variabilidad fue superior a aquella reportada por Behera y Shukla (2015) y Deressa et al. (2013) para $\mathrm{Ca}, \mathrm{K}$ y Mg. Debe recordarse, que las muestras evaluadas correspondieron a todo el territorio Peruano.

Las concentraciones de $\mathrm{H}$ en la Tabla 2 se obtuvieron de la diferencia entre la acidez extractable y la concentración de $\mathrm{Al}$ extractable. Este método produce valores generalmente mayores que aquellos obtenidos a partir del pH del extracto de $\mathrm{KCl} 1 \mathrm{M}$ (Ross et al., 1996). En este estudio, se confirmó esta observación. Aparte de $\mathrm{H}^{+}$soluble e intercambiable, la solución de $\mathrm{KCl} 1 \mathrm{M}$ también extrae sustancias inorgánicas y orgánicas diversas que pueden liberar $\mathrm{H}^{+}$adicional durante la titulación de la acidez extractable. Por lo tanto, el método potenciométrico parece ser más apropiado y recomendable para evaluar el $\mathrm{H}$ extractable o intercambiable en muestras de suelo. 
Lasumadelas concentraciones de elementos extractables de los suelos estudiados presentó un valor promedio igual a $5,85 \mathrm{cmol} \mathrm{kg}^{-1}$ y un coeficiente de variabilidad de $113 \%$. Aunque esta no se expresó en términos de carga eléctrica, constituye una manera alternativa para definir la capacidad de intercambio catiónico efectiva para una muestra de suelo. No se encontraron valores comparables en la literatura revisada.

Las fracciones molares extractables promedio de elementos en las muestras de suelos ácidos del Perú disminuyeron en el orden: $\mathrm{Al}>\mathrm{Ca}>\mathrm{K}>\mathrm{Mg}>\mathrm{Na}>\mathrm{H}>$ $\mathrm{Mn}>\mathrm{Fe}>\mathrm{Zn}>\mathrm{Cu}$. En conjunto, $\mathrm{Al}$ y $\mathrm{Ca}$ constituyeron $70 \%$ de la concentración total media de elementos

Tabla 2. Valores reportados de acidez extractable $\left(\mathrm{cmol} \mathrm{H}^{+} \mathrm{kg}^{-1}\right)$ y concentraciones de elementos intercambiables $\left(\mathrm{cmol} \mathrm{kg}^{-1}\right)$ para suelos ácidos del mundo

\begin{tabular}{ccl}
\hline Sustancia & Rango & \multicolumn{1}{c}{ Referencias Bibliográficas* } \\
\hline $\begin{array}{c}\text { Acidez } \\
\text { Extractable }\end{array}$ & $0,08-11$ & $4,12,18$ \\
\hline $\mathrm{Al}$ & $<0,003-14$ & $1,2,4,6,7,8,11,14,16,17,18$ \\
$\mathrm{Ca}$ & $<0,005-17$ & $1,2,3,5,6,8,9,11,12,13,14,15,16,17,18$ \\
$\mathrm{Cu}$ & $0,002-0,092$ & $7,10,16,19$ \\
$\mathrm{Fe}$ & $<0,01-0,53$ & $2,6,8,16$ \\
$\mathrm{H}$ & $0,08-20$ & $8,11,14,17$ \\
$\mathrm{~K}$ & $0,01-3,8$ & $1,2,3,5,6,8,9,11,12,13,14,15,18$ \\
$\mathrm{Mg}$ & $0,01-8,6$ & $1,2,3,5,6,8,9,11,12,13,14,16,18$ \\
$\mathrm{Mn}$ & $0,07-0,35$ & $2,6,7,16,19$ \\
$\mathrm{Na}$ & $0,03-3,4$ & $1,2,6,8,11,15,16$ \\
$\mathrm{Zn}$ & $0,0003-0,072$ & $7,10,16,19$
\end{tabular}

* 1: James et al. (2016); 2: Shi et al. (2016); 3: Behera y Shukla (2015); 4: Hengl et al. (2015); 5: Kim et al. (2015); 6: Alling et al. (2014); 7: Arenas-Lago et al. (2014); 8: Lu et al. (2014); 9: Pérez-Esteban et al. (2014a); 10: Pérez-Esteban et al. (2014b); 11: Asensio et al. (2013); 12: Deressa et al. (2013); 13: Tomasic et al. (2013); 14: Van der Heijden et al. (2013); 15 Yitbarek et al. (2013); 16: Stevens et al. (2009); 17: Ross et al. (1996); 18: Lopes y Cox (1977); 19: Dolar y Keeney (1971)

Tabla 3. Coeficientes de correlación lineal simple de contenido de materia orgánica $(\mathrm{MO}, \%)$, contenido de arcilla (\%) y $\mathrm{pH} 1: 1$ en agua con acidez extractable $\left(\mathrm{cmol} \mathrm{H} \mathrm{Hg}^{-1}\right)$ y las concentraciones $\left(\mathrm{cmol} \mathrm{kg}^{-1}\right)$ y fracciones molares $\left(\mathrm{mol} \mathrm{mol}^{-1}\right)$ de elementos extractables en muestras de suelos ácidos del Perú

\begin{tabular}{ccccccc}
\hline \multirow{2}{*}{ Sustancia } & \multicolumn{3}{c}{ Cantidad Extractable } & \multicolumn{3}{c}{ Fracción Molar Extractable } \\
\cline { 2 - 7 } & $\mathrm{MO}$ & Arcilla & $\mathrm{pH}$ & $\mathrm{MO}$ & Arcilla & $\mathrm{pH}$ \\
\hline Acidez Extractable & $-0,02$ & $-0,05$ & $-0,53^{*}$ & - & - & - \\
$\mathrm{Al}$ & $-0,07$ & 0,03 & $-0,35^{*}$ & $-0,23^{*}$ & 0,00 & $-0,42^{*}$ \\
$\mathrm{Ca}$ & 0,13 & 0,19 & 0,16 & 0,18 & 0,13 & $0,50^{*}$ \\
$\mathrm{Cu}$ & 0,15 & $-0,12$ & $-0,31^{*}$ & 0,15 & $-0,16$ & $-0,28^{*}$ \\
$\mathrm{Fe}$ & $-0,03$ & $-0,11$ & $-0,36^{*}$ & 0,00 & $-0,12$ & $-0,39^{*}$ \\
$\mathrm{H}$ & $0,35^{*}$ & $-0,17$ & $-0,67^{*}$ & 0,13 & $-0,22^{*}$ & $-0,64^{*}$ \\
$\mathrm{~K}$ & $0,34^{*}$ & 0,07 & 0,10 & 0,08 & $-0,20$ & $-0,02$ \\
$\mathrm{Mg}$ & 0,06 & 0,19 & $0,24^{*}$ & 0,02 & 0,03 & $0,32^{*}$ \\
$\mathrm{Mn}$ & 0,16 & $-0,04$ & 0,02 & $-0,03$ & $-0,21^{*}$ & 0,15 \\
$\mathrm{Na}$ & 0,12 & $0,24^{*}$ & $0,23^{*}$ & $-0,14$ & $-0,06$ & 0,09 \\
$\mathrm{Zn}$ & $0,27^{*}$ & $-0,14$ & $-0,36^{*}$ & $0,30^{*}$ & $-0,15$ & $-0,37^{*}$ \\
Suma Elementos & 0,16 & 0,17 & $-0,04$ & - & - & - \\
\hline
\end{tabular}

Nota: Valores marcados con $*$ son estadísticamente diferentes a 0,00 para un nivel de significación igual a 0,05 .
Distribución de los datos en función del $\mathrm{pH}$

Las concentraciones de sustancias extractables en las muestras de suelos ácidos y sus fracciones molares presentaron mejor correlación con el $\mathrm{pH}$ que con los contenidos de materia orgánica y arcilla, como se ilustra en la Tabla 3. Por ello, la diversidad de los datos de este estudio se presentó en función del $\mathrm{pH}$. Cabe notar, que se utilizó el valor límite de $0,5 \mathrm{dS} \mathrm{m}^{-1}$ para diferenciar muestras de baja y alta conductividad eléctrica.

La acidez extractable de las muestras estudiadas en función del pH se muestra en la Figura 1. Los valores más pequeños de acidez extractable fueron observados en el rango de $\mathrm{pH}$ de 5 a 6 . A medida que disminuyó el $\mathrm{pH}$, la acidez extractable aumentó en magnitud y amplitud. Ambos parámetros tuvieron una correlación lineal significativa, como indicado en la Tabla 3. La alta correlación fue esperada, ya que un menor pH está asociado con un medio mas ácido. Esta tendencia ha sido reportada en investigaciones, tales como la de Deressa et al. (2013).

La relación entre la suma de las concentraciones deelementos extractables para las muestras de suelos ácidos del Perú en relación con el $\mathrm{pH}$ también se muestra en la Figura 1. En términos globales, estos parámetros no estuvieron correlacionados significativamente, como puede observarse en la Tabla 3 . Sin embargo, para el grupo de muestras de baja conductividad eléctrica, esta relación presentó un coeficiente de correlación lineal simple de $+0,34$, que resultó significativo. Para las muestras de alta conductividad eléctrica, este coeficiente tuvo un valor igual a $-0,22$ y no alcanzó significación estadística. 

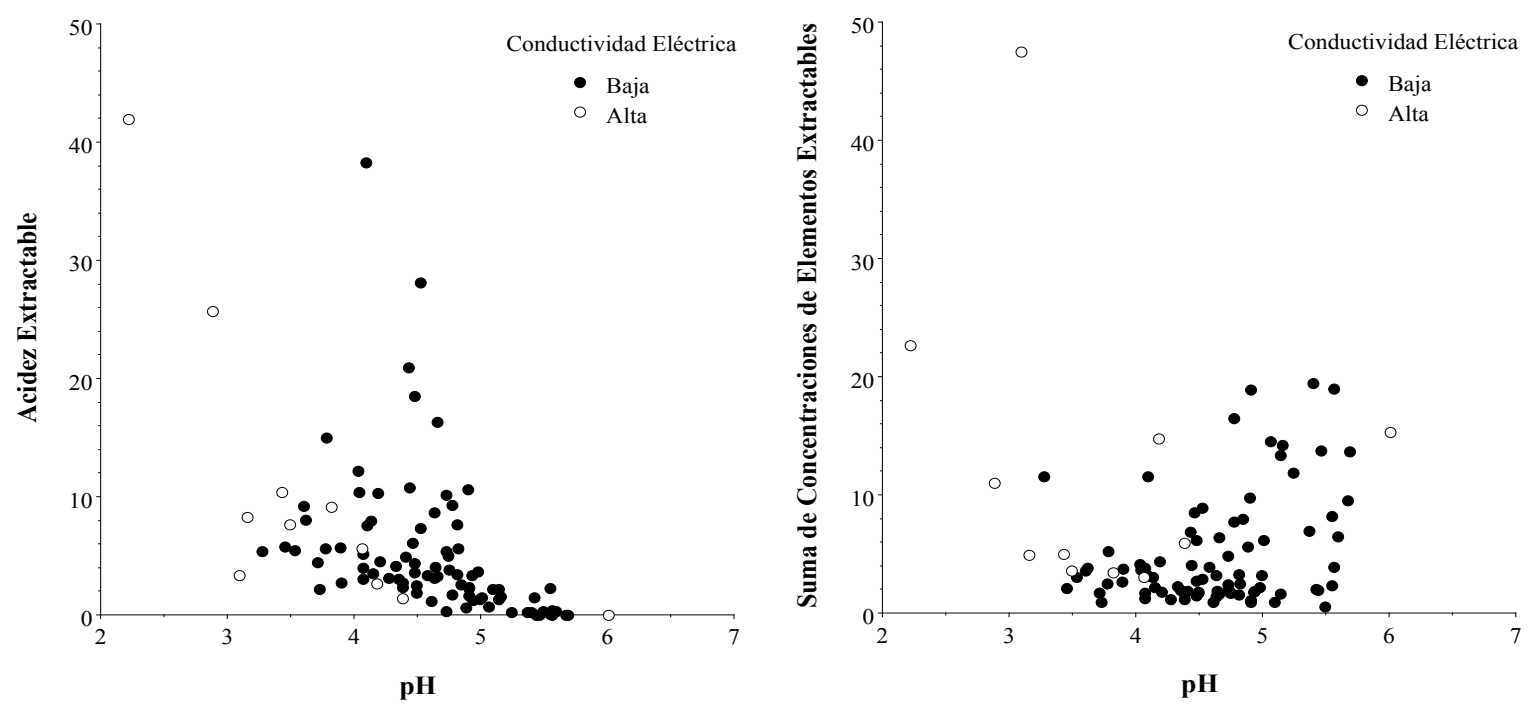

Figura 1. Relaciones de la acidez extractable $\left(\mathrm{cmol} \mathrm{H}^{+} \mathrm{kg}^{-1}\right)$ y la suma de concentraciones de elementos extractables $\left(\mathrm{cmol} \mathrm{kg}^{-1}\right)$ con el $\mathrm{pH}$ en muestras de suelos ácidos del Perú
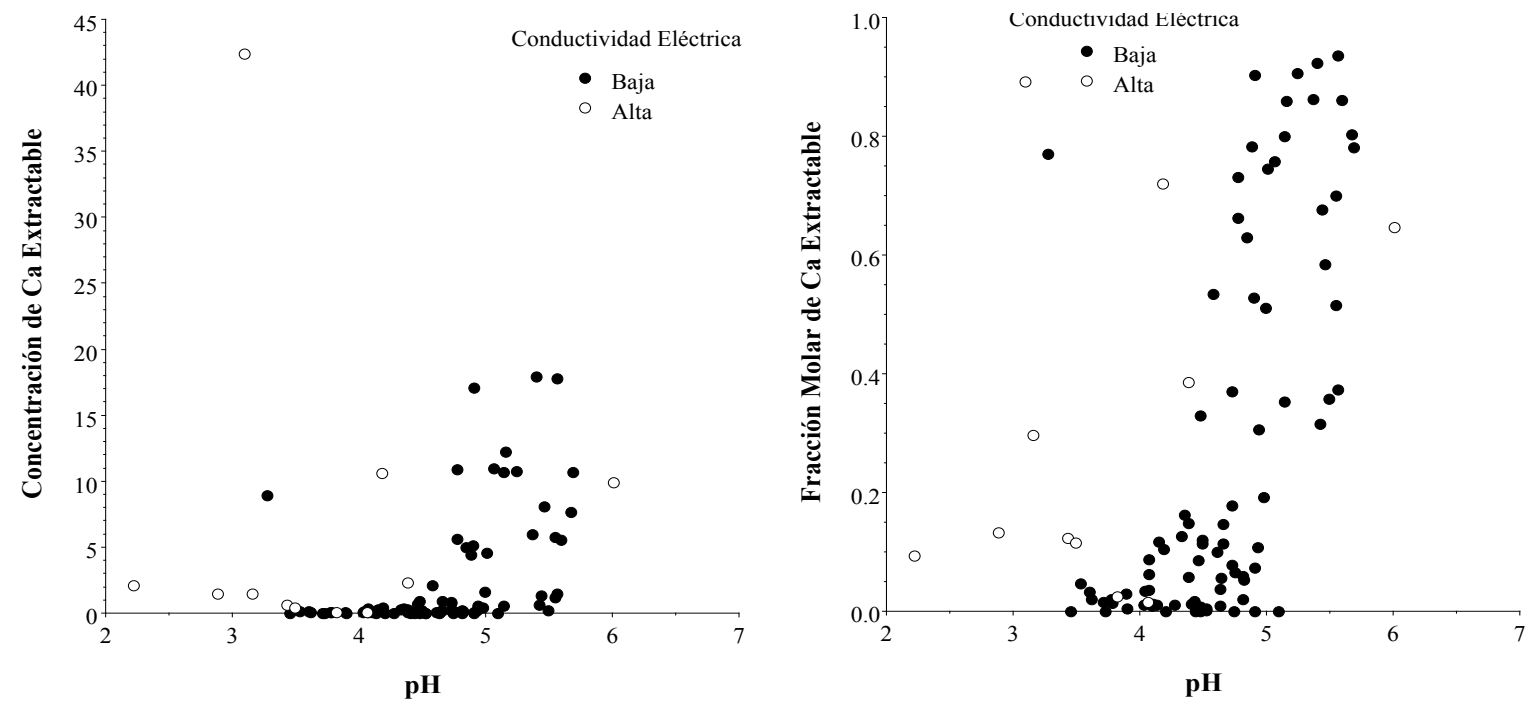

Figura 2. Relaciones de la concentración $\left(\mathrm{cmol} \mathrm{kg}^{-1}\right)$ y fracción molar $\left(\mathrm{mol} \mathrm{mol}^{-1}\right)$ de Ca extractable con el $\mathrm{pH}$ en muestras de suelos ácidos del Perú

Correlaciones positivas entre el $\mathrm{pH}$ y las concentraciones de $\mathrm{Ca}, \mathrm{K}, \mathrm{Mg}$ y $\mathrm{Na}$ intercambiables han sido comúnmente reportadas (Behera y Shukla, 2015; Deressa et al., 2013; Lu et al., 2014; Stevens et al., 2009). Las relaciones del $\mathrm{pH}$ con las concentraciones y fracciones molares extractables de $\mathrm{Ca}, \mathrm{K}, \mathrm{Mg}, \mathrm{Mn}$ y $\mathrm{Na}$ obtenidas en este estudio se ilustran en las Figuras 2, 3, 4, 5 y 6, respectivamente. La examinación rápida de estos gráficos sugiere que el pH tendió a estar directamente relacionado solo con la concentración extractable de Na y las fracciones molares de Mg, Mn y Na. En estos casos, los valores de las variables dependientes tendieron a ser pequeños y mostrar poca variabilidad en el rango de $\mathrm{pH}$ de 2 a 3 . Sin embargo, al aumentar el pH se incrementó la ocurrencia de valores cada vez mas altos y la variabilidad de los datos, dando la apariencia de un abanico.
Correlación significativa del $\mathrm{pH}$ con la concentración y la fracción molar extractables solamente se observó para el $\mathrm{Mg}$, como indicado en la Tabla 3. Para el Ca, únicamente la fracción molar resultó significativa. Las muestras con baja conductividad eléctrica mostraron mejores relaciones del $\mathrm{pH}$ con estas dos variables. Así, los coeficientes de correlación lineal alcanzaron valores significativos de $+0,47$ y $+0,64$, respectivamente. El $\mathrm{pH}$ no estuvo significativamente correlacionado con la concentración y la fracción molar extractables de K y Mn. Al excluir las muestras de alta conductividad eléctrica del análisis, las relaciones del $\mathrm{H}$ con ambas variables solo mejoraron para el Mn. Para este, los coeficientes de correlación lineal fueron iguales a $+0,35$ y $+0,20$, respectivamente, y solamente el primero resultó significativo. En contraste, los valores correspondientes para las muestras de alta conductividad eléctrica fueron de $-0,39$ y $-0,33$, respectivamente, y 
carecieron de significación estadística. En el caso del $\mathrm{Na}$, el pH tuvo una correlación significativa solo con la concentración extractable. En el grupo de muestras de alta conductividad eléctrica, los coeficientes de correlación lineal del $\mathrm{pH}$ con la concentración y la fracción molar extractables de $\mathrm{Na}$ fueron significativas e iguales a $+0,81$ y $+0,92$, respectivamente.

El pH mostró una relación inversa con las concentraciones y fracciones molares extractables de Al, $\mathrm{Cu}, \mathrm{Fe}, \mathrm{H}$ y Zn, como ilustrado en las Figuras 7, 8, 9, 10 y 11, respectivamente. En estas figuras, los valores de las variables dependientes tendieron a ser pequeños y mostrar poca variabilidad en el rango de $\mathrm{pH}$ de 5 a 6 . Sin embargo, al disminuir el $\mathrm{pH}$ aumentó la ocurrencia de valores cada vez más altos y se incrementó la variabilidad de los datos, dando la apariencia de un abanico. A valores de $\mathrm{pH}$ alrededor de 4,5, algunas muestras alcanzaron fracciones molares de $\mathrm{Al}$ superiores a 0,9 , mientras que otras tuvieron valores cercanos a 0 . Correlaciones negativas entre el
$\mathrm{pH}$ y las concentraciones de $\mathrm{Al}$ e $\mathrm{H}$ intercambiables han sido reportadas comúnmente (Deressa et al., 2013; Ross et al., 1996; Sims, 1996). La relación inversa entre $\mathrm{pH}$ y concentración de $\mathrm{Fe}$ intercambiable también ha sido previamente documentada (Stevens et al., 2009). La solubilidad de Al y Fe típicamente tiende a aumentar al incrementar la acidez del suelo (Lindsay, 1979).

Correlación significativa del $\mathrm{pH}$ con la concentración y la fracción molar extractables se observó para $\mathrm{Al}, \mathrm{Cu}, \mathrm{Fe}$, $\mathrm{H}$ y $\mathrm{Zn}$, como mostrado en la Tabla 3. De este grupo, el H tuvo el mayor valor de coeficiente de correlación lineal. Por otra parte, la concentración de $\mathrm{H}$ extractable (HE, cmol $\mathrm{kg}^{-1}$ ) presentó la relación más clara y menos variable con el pH del suelo. Esta relación pudo descibirse mediante la ecuación

$$
\mathrm{HE}=21.86 \cdot 10^{-0.5605} \mathrm{pH}
$$

siendo el coeficiente de determinación igual a 0,743.
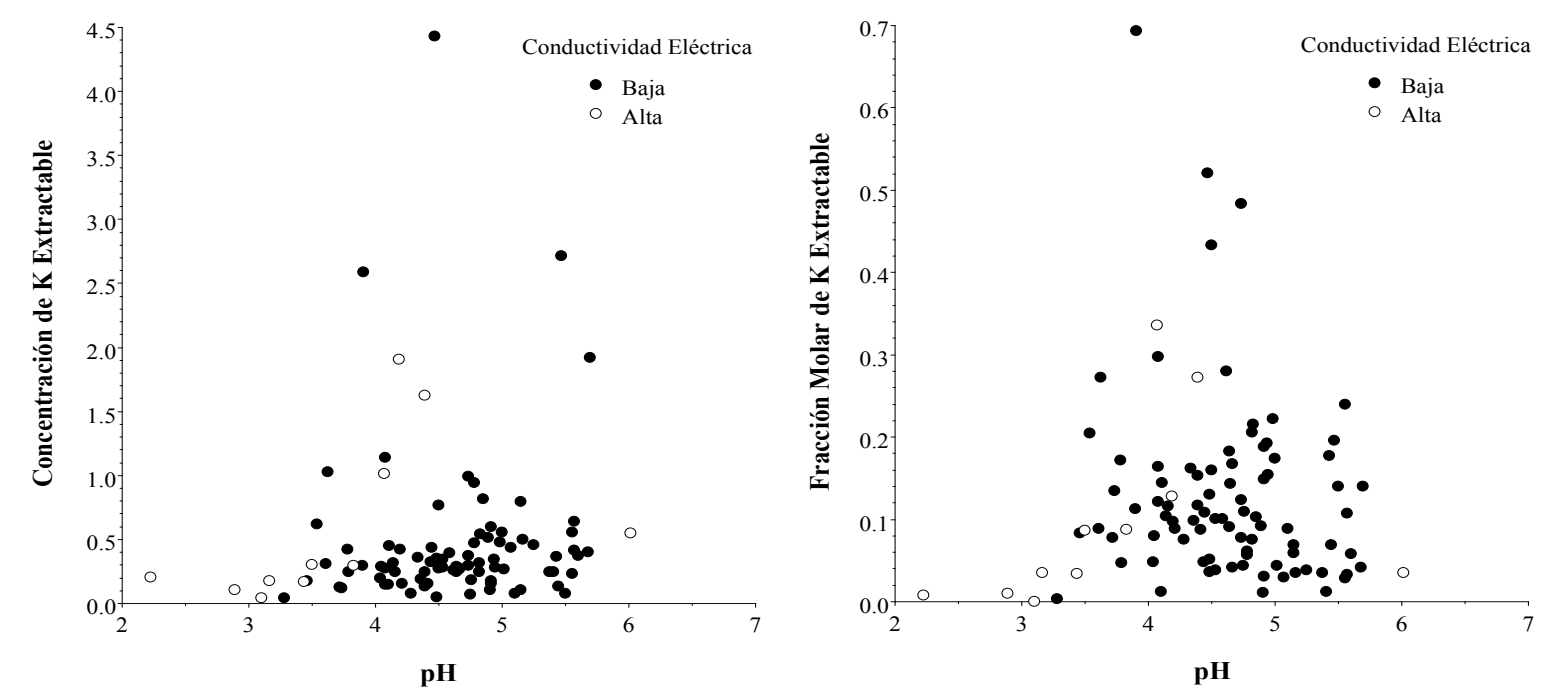

Figura 3. Relaciones de la concentración $\left(\mathrm{cmol} \mathrm{kg}^{-1}\right)$ y fracción molar $\left(\mathrm{mol} \mathrm{mol}^{-1}\right)$ de $\mathrm{K}$ extractable con el pH en muestras de suelos ácidos del Perú
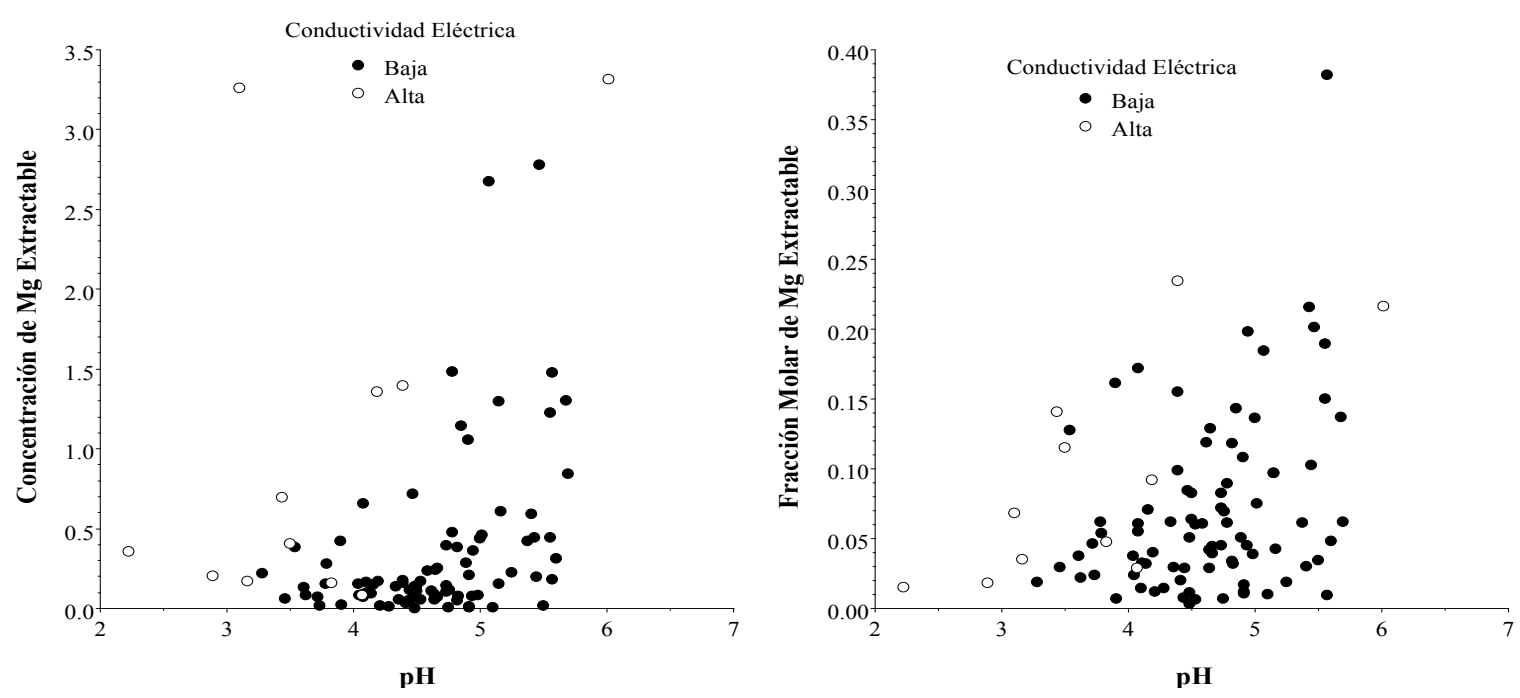

Figura 4. Relaciones de la concentración $\left(\mathrm{cmol} \mathrm{kg}^{-1}\right)$ y fracción molar $\left(\mathrm{mol} \mathrm{mol}^{-1}\right)$ de $\mathrm{Mg}$ extractable con el pH en muestras de suelos ácidos del Perú 

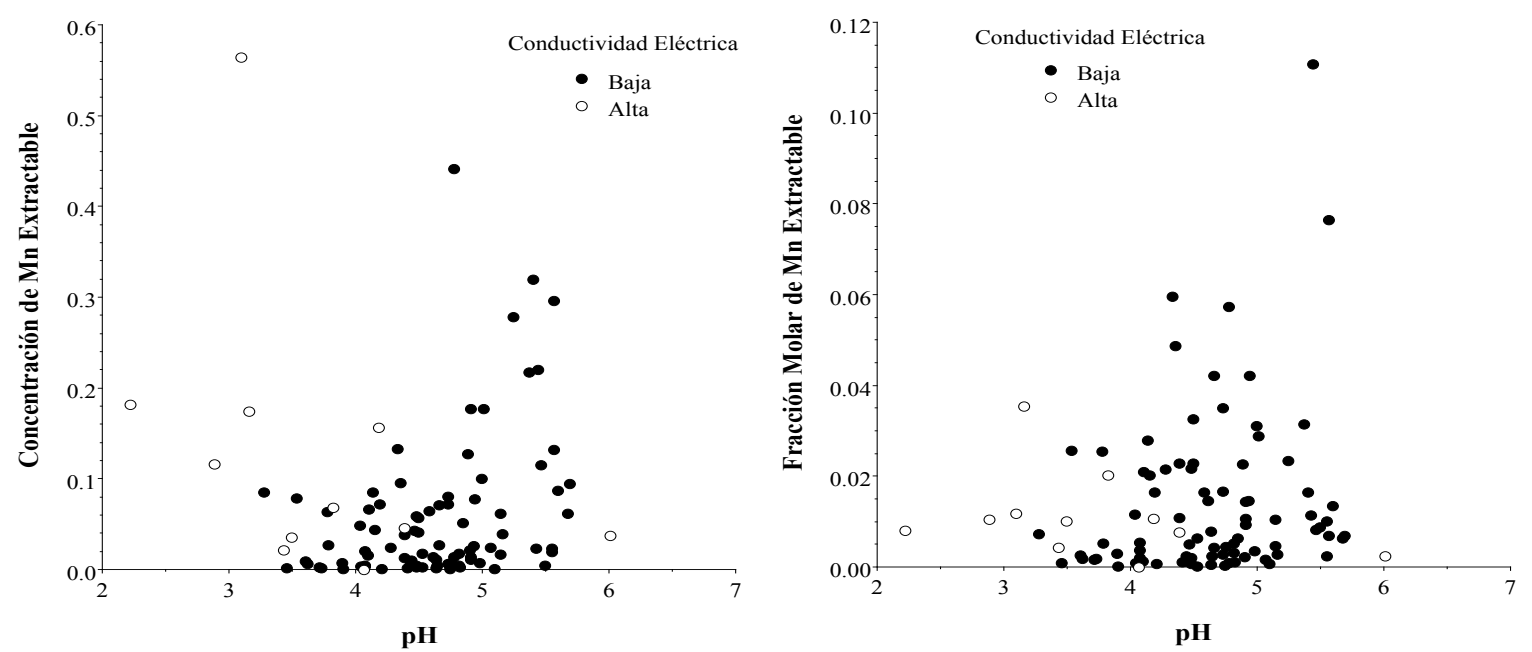

Figura 5. Relaciones de la concentración $\left(\mathrm{cmol} \mathrm{kg}^{-1}\right)$ y fracción molar $\left(\mathrm{mol} \mathrm{mol}^{-1}\right)$ de Mn extractable con el $\mathrm{pH}$ en muestras de suelos ácidos del Perú
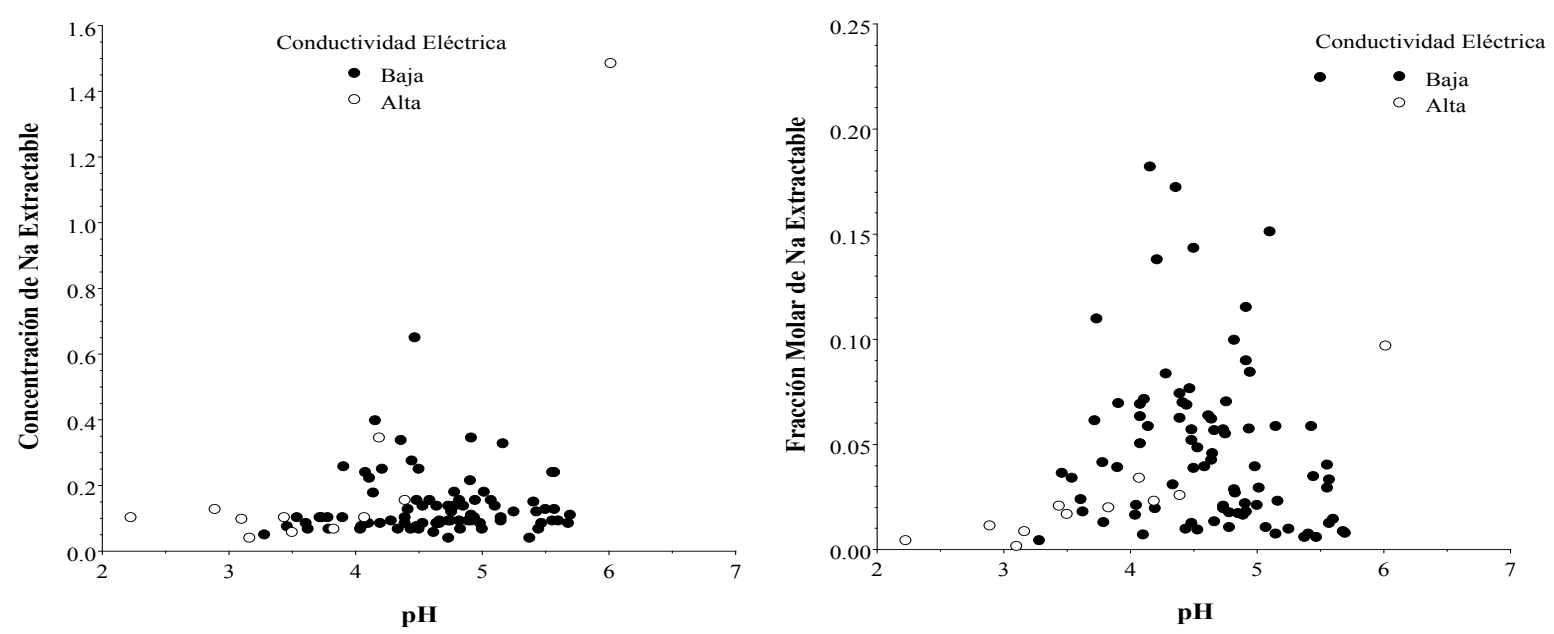

Figura 6. Relaciones de la concentración $\left(\mathrm{cmol} \mathrm{kg}^{-1}\right)$ y fracción molar $\left(\mathrm{mol} \mathrm{mol}^{-1}\right)$ de Na extractable con el pH en muestras de suelos ácidos del Perú
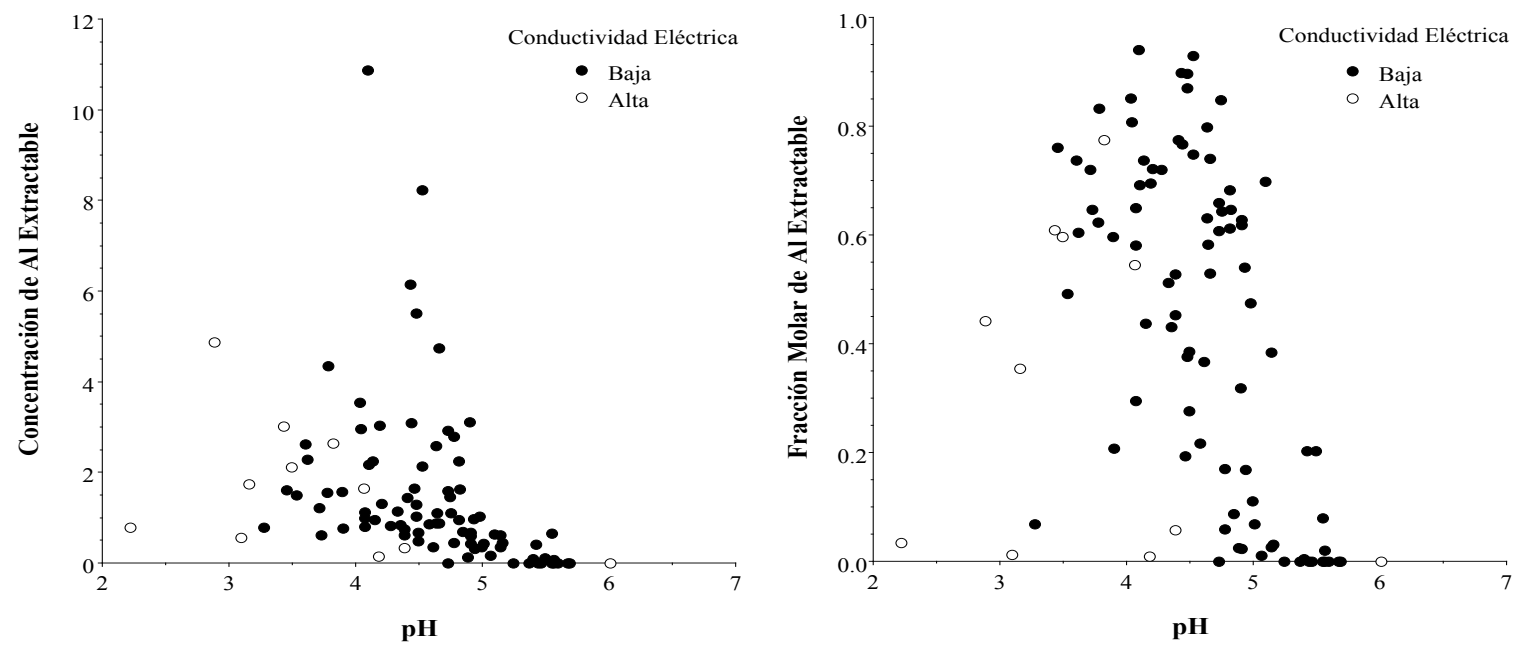

Figura 7. Relaciones de la concentración $\left(\mathrm{cmol} \mathrm{kg}^{-1}\right)$ y fracción molar $\left(\mathrm{mol} \mathrm{mol}^{-1}\right)$ de $\mathrm{Al}$ extractable con el pH en muestras de suelos ácidos del Perú 

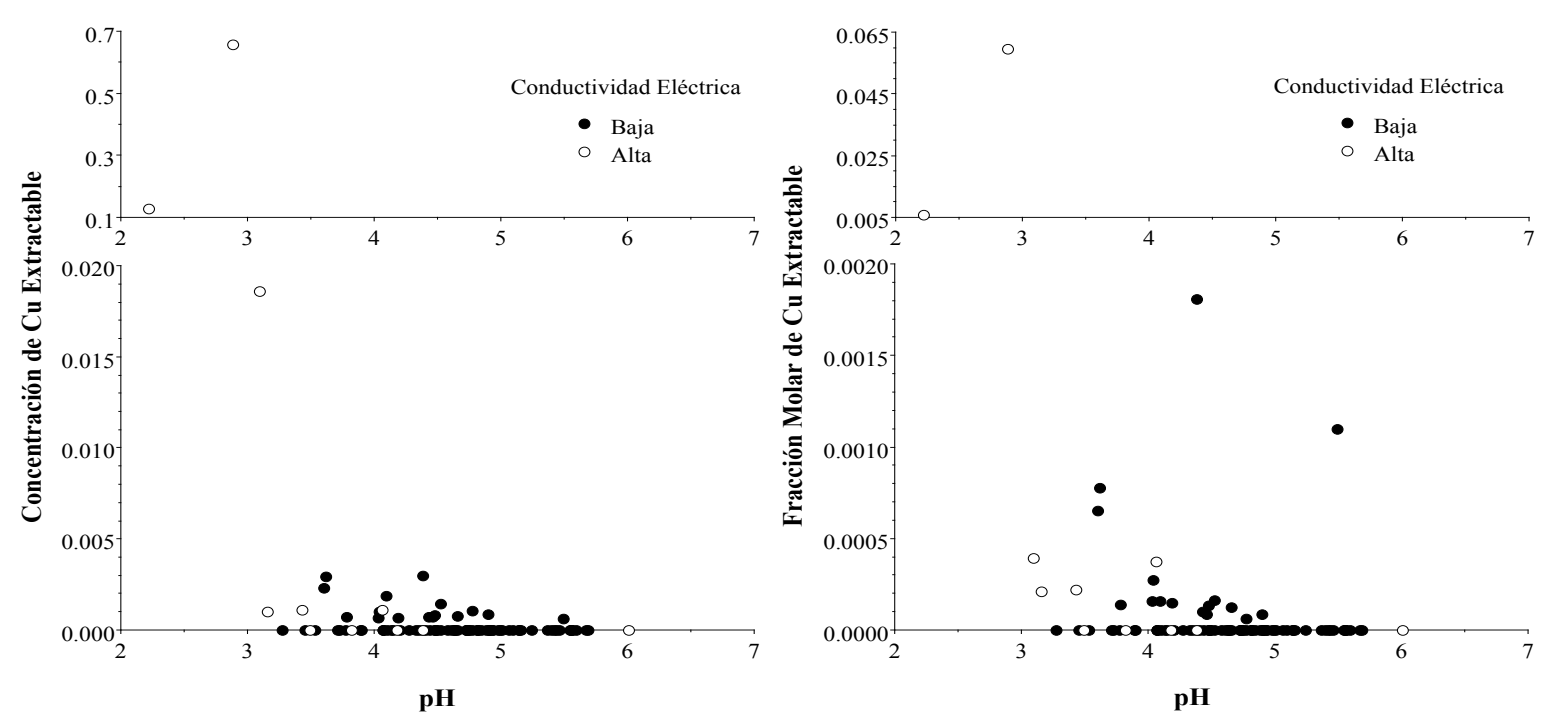

Figura 8. Relaciones de la concentración $\left(\mathrm{cmol} \mathrm{kg}^{-1}\right)$ y fracción molar $\left(\mathrm{mol} \mathrm{mol}{ }^{-1}\right)$ de $\mathrm{Cu}$ extractable con el $\mathrm{pH}$ en muestras de suelos ácidos del Perú
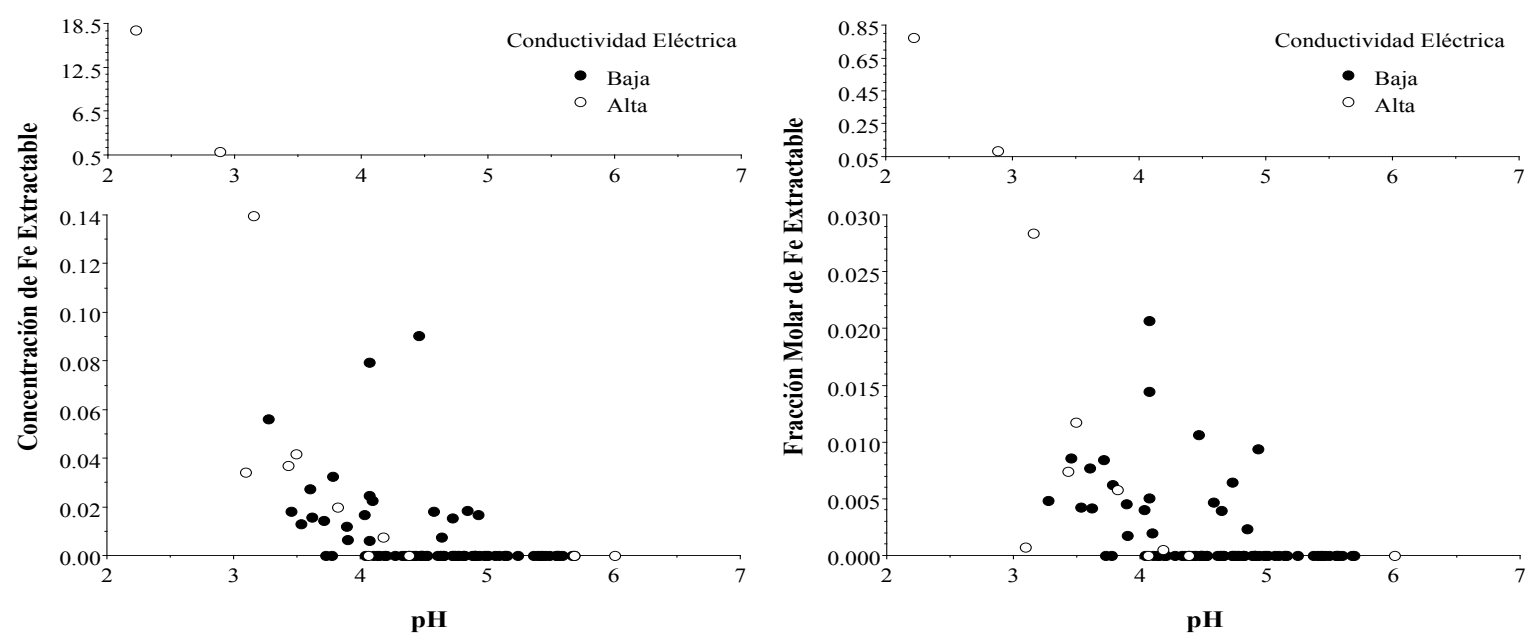

Figura 9. Relaciones de la concentración $\left(\mathrm{cmol} \mathrm{kg}^{-1}\right)$ y fracción molar $\left(\mathrm{mol} \mathrm{mol}^{-1}\right)$ de Fe extractable con el $\mathrm{pH}$ en muestras de suelos ácidos del Perú
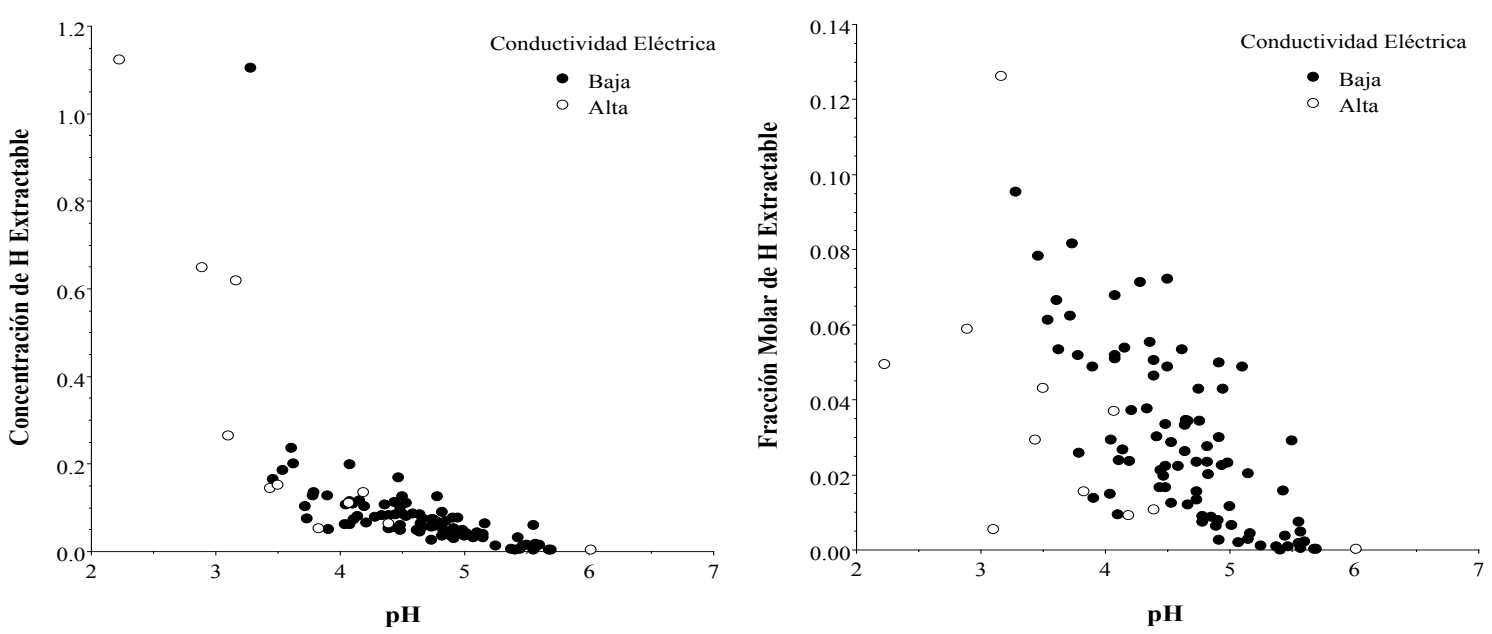

Figura 10. Relaciones de la concentración $\left(\mathrm{cmol} \mathrm{kg}^{-1}\right)$ y fracción molar $\left(\mathrm{mol} \mathrm{mol}^{-1}\right)$ de $\mathrm{H}$ extractable con el $\mathrm{pH}$ en muestras de suelos ácidos del Perú 

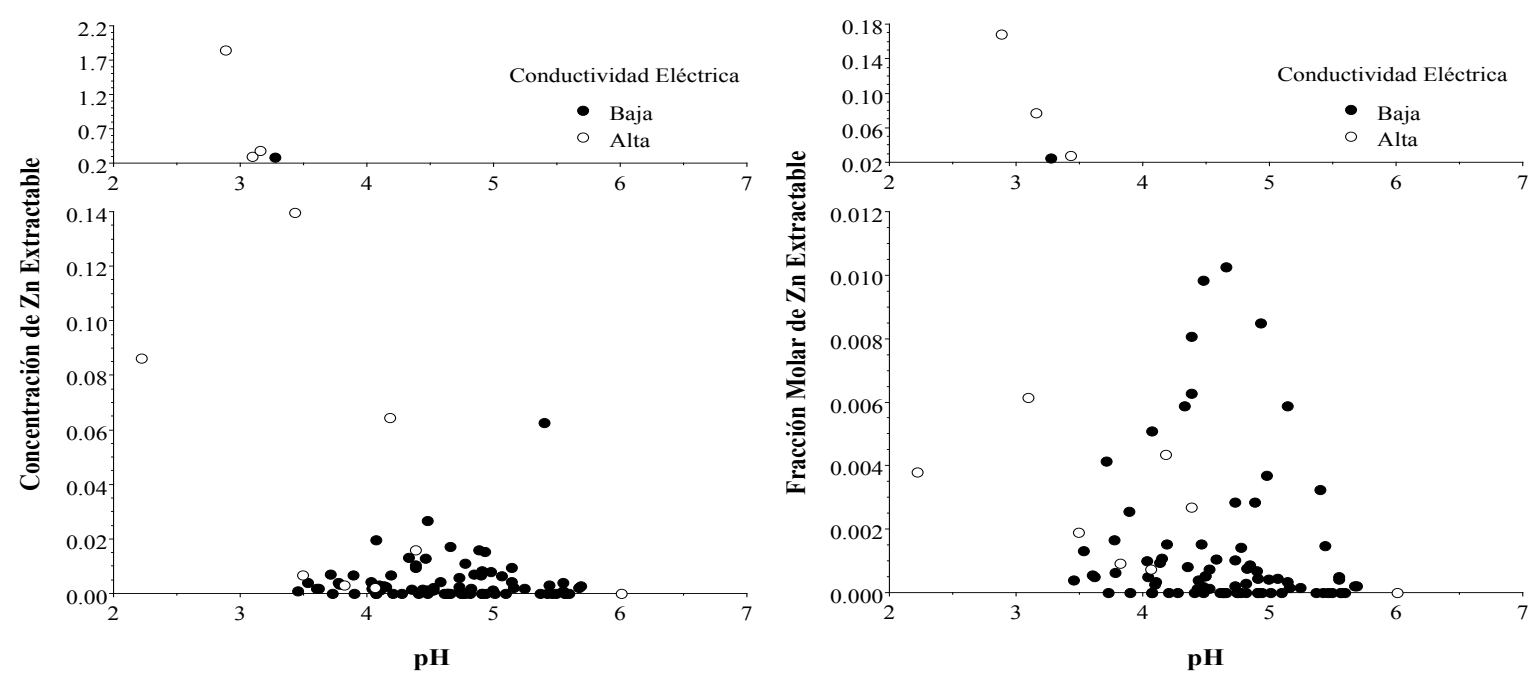

Figura 11. Relaciones de la concentración $\left(\mathrm{cmol} \mathrm{kg}^{-1}\right)$ y fracción molar $\left(\mathrm{mol} \mathrm{mol}^{-1}\right)$ de $\mathrm{Zn}$ extractable con el $\mathrm{pH}$ en muestras de suelos ácidos del Perú

Comparativo de muestras de alta y baja fracción soluble extractable

Las soluciones de $\mathrm{KCl} 1 \mathrm{M}$ y $\mathrm{CH}_{3} \mathrm{COONH}_{4} 1 \mathrm{M} \mathrm{pH} 7$ extraen las porciones soluble en agua, intercambiable y disuelta de fase sólida de elementos. La porción soluble en agua puede constituir una parte significativa del total extractable. Esta porción se puede excluir mediante lavado previo de la muestra con agua o substracción matemática de su magnitud determinada independientemente. Alternativamente, en muestras de baja conductividad eléctrica, las concentraciones extractables deberían contener predominantemente la suma de las porciones intercambiable y disuelta de fase sólida de los elementos. La magnitud de la segunda de estas no se puede diferenciar con facilidad.

La estadística descriptiva para muestras de baja $(<0,5$ $\left.\mathrm{dS} \mathrm{m}^{-1}\right)$ y alta $\left(\geq 0,5 \mathrm{dS} \mathrm{m}^{-1}\right)$ conductividad eléctrica de los suelos ácidos estudiados se ilustra en la Figura 12. Los números de observaciones para estos grupos fueron iguales a 85 y 11 , respectivamente.

Enelanálisis del grupodemuestras de baja conductividad eléctrica, las concentraciones medias de elementos extractables disminuyeron en el orden: $\mathrm{Ca}>\mathrm{Al}>\mathrm{K}>\mathrm{Mg}>$ $\mathrm{Na}>\mathrm{H}>\mathrm{Mn}>\mathrm{Zn}>\mathrm{Fe}>\mathrm{Cu}$. Este ordenamiento también sería válido para las concentraciones intercambiable más disuelta de fase sólida en las muestras de suelo. Esta secuencia fue similar a la obtenida en el análisis de todas las muestras, excepto por el Fe. Las fracciones molares promedio de elementos extractables para este grupo de suelos de baja conductividad eléctrica decrecieron en el orden: $\mathrm{Al}>\mathrm{Ca}>\mathrm{K}>\mathrm{Mg}>\mathrm{Na}>\mathrm{H}>\mathrm{Mn}>\mathrm{Fe}>\mathrm{Zn}$ $>\mathrm{Cu}$, siendo el ordenamiento de los elementos igual que en el análisis de todas las muestras. En promedio, Al y $\mathrm{Ca}$ en las formas intercambiable y disuelta de fase sólida conjuntamente constituyeron $70 \%$ de la concentración total de elementos. Al adicionar las contribuciones de $\mathrm{K}$ y Mg, se alcanzó el $90 \%$ del total. Estos valores también fueron similares a aquellos obtenidos en el análisis de todas las muestras.

En el grupo de muestras de alta conductividad eléctrica, las concentraciones promedio de elementos extractables decrecieron en el orden: $\mathrm{Ca}>\mathrm{Fe}>\mathrm{Al}>\mathrm{Mg}>\mathrm{K}>\mathrm{H}>$ $\mathrm{Zn}>\mathrm{Na}>\mathrm{Mn}>\mathrm{Cu}$. Esta secuencia difiere claramente de aquella para muestras de baja conductividad eléctrica. El Fe superó a Zn, Mn, H, Na, Mg, K y Al; el Zn, a Mn y Na; el H, a Na; y el Mg, a K. El ordenamiento respectivo para las fracciones molares extractables promedio fue: $\mathrm{Al}=\mathrm{Ca}$ $>\mathrm{K}>\mathrm{Mg}>\mathrm{Fe}>\mathrm{H}>\mathrm{Zn}>\mathrm{Na}>\mathrm{Mn}>\mathrm{Cu}$. Este orden también difiere, aunque menos que las muestras de baja conductividad eléctrica. En este caso, el Fe superó a Mn, $\mathrm{H}$ y Na; el Zn, a Mn y Na; y el H, a Na. En ambas secuencias, $\mathrm{Fe}$ y $\mathrm{Zn}$ fueron los elementos de mayor variación. Además, Al y Ca solo lograron representar el $63 \%$ de la suma de concentraciones extractables de los elementos estudiados. Al agregar K y Mg, se llegó a $81 \%$ del total. Cabe notar, que la mitad de estas muestras tiene $\mathrm{pH}$ menor que 3,5 , y algunas de estas provienen de suelos sulfatados ácidos. Finalmente, este grupo incluye a las muestras de características extremas que presentan los mayores valores de suma de concentraciones de elementos extractables, acidez extractable y concentraciones extractables de $\mathrm{Ca}$, $\mathrm{Cu}, \mathrm{Fe}, \mathrm{H}, \mathrm{Mn}$ y $\mathrm{Zn}$. Así, la secuencia de abundancia de los elementos puede diferir notablemente cuando una parte importante de la concentración extractable está presente en forma soluble en agua.

\section{Conclusiones}

En las 96 muestras de suelos ácidos del Perú, las concentraciones extractables medias decrecieron en el orden $\mathrm{Ca}>\mathrm{Al}>\mathrm{K}>\mathrm{Mg}>\mathrm{Fe}>\mathrm{Na}>\mathrm{H}>\mathrm{Mn}>\mathrm{Zn}>\mathrm{Cu}$, mientras que las fracciones molares extractables promedio 

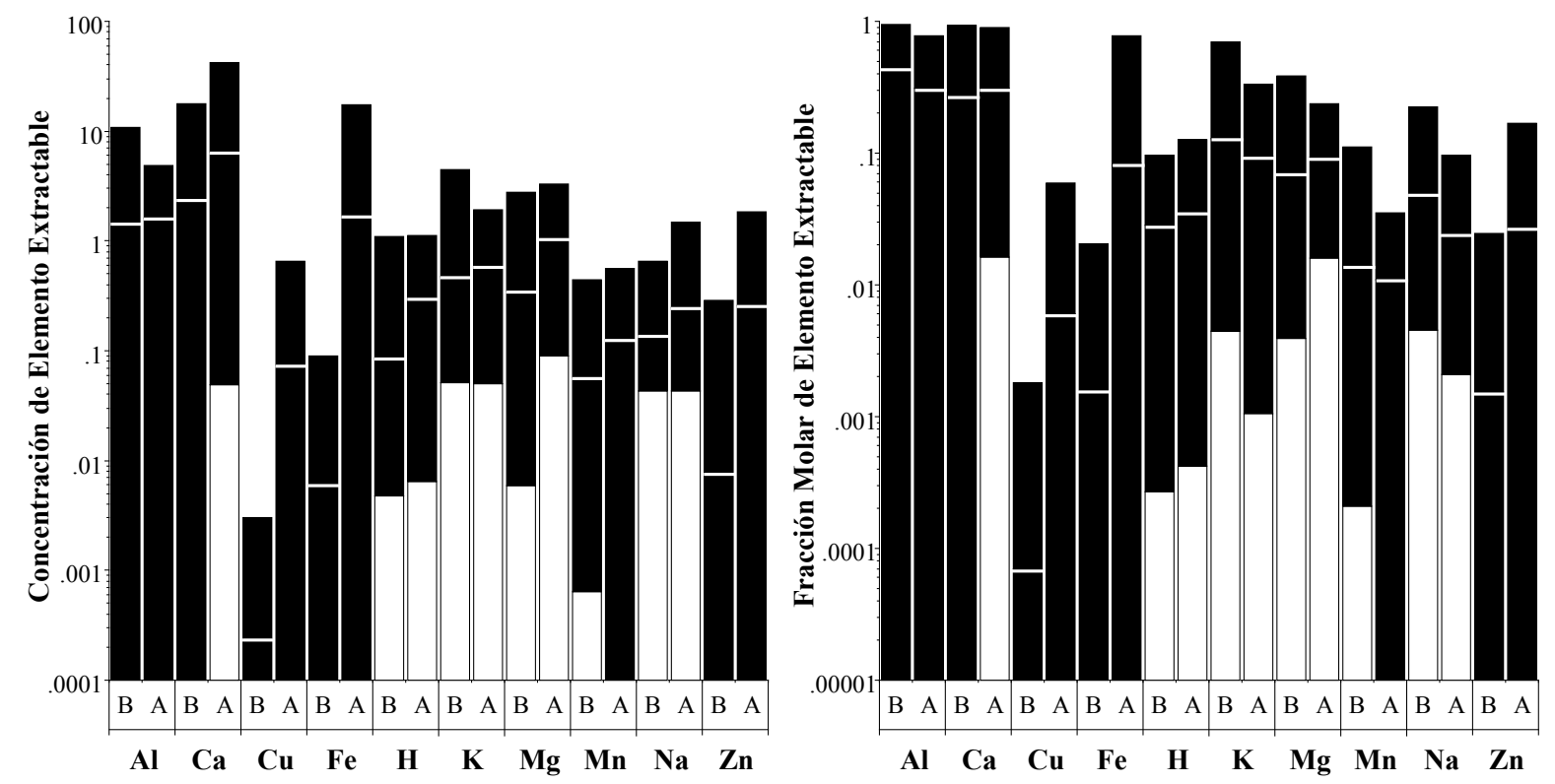

Figura 12. Rangos (barras negras) y medias (líneas blancas) de concentración ( $\mathrm{cmol} \mathrm{kg}^{-1}$ ) y fracción molar ( $\left.\mathrm{mol} \mathrm{mol}{ }^{-1}\right)$ de elementos extractables en muestras de suelos ácidos del Perú, agrupadas en baja (B) y alta (A) conductividad eléctrica

disminuyeron en la secuencia $\mathrm{Al}>\mathrm{Ca}>\mathrm{K}>\mathrm{Mg}>\mathrm{Na}>\mathrm{H}$ $>\mathrm{Mn}>\mathrm{Fe}>\mathrm{Zn}>\mathrm{Cu}$. En promedio, las concentraciones de $\mathrm{Al}, \mathrm{Ca}, \mathrm{K}$ y $\mathrm{Mg}$ sumaron $90 \%$ del total. El elemento predominante en los extractos de muestras de suelo muy fuertemente ácidas fue $\mathrm{Al}$; y en muestras medianamente ácidas, el $\mathrm{Ca}$. El rango de $\mathrm{pH}$ para la transición respectiva fue 4,5 a 5,0. El pH estuvo directamente relacionado con el contenido de $\mathrm{Mg}$ y, en orden decreciente, con elementos como Ca y $\mathrm{Na}$. Por otra parte, el $\mathrm{pH}$ presentó una relación inversa con los contenidos de $\mathrm{Al}, \mathrm{Cu}, \mathrm{Fe}, \mathrm{H}$ y $\mathrm{Zn}$, siendo esta relación más clara para el $\mathrm{H}$. Dentro del grupo de muestras con una parte importante de la cantidad extractable en forma soluble en agua, se encontraron aquellas con $\mathrm{pH}$ menor que 3 o sea las muestras de suelo extremadamente ácidas y con mayores contenidos de $\mathrm{Cu}, \mathrm{Fe}$ y $\mathrm{Zn}$.

\section{Agradecimientos}

El autor agradece al Laboratorio de Análisis de Suelos, Plantas, Aguas y Fertilizantes de la Facultad de Agronomía de la Universidad Nacional Agraria La Molina por darle las facilidades necesarias para realizar ciertos análisis y ayudar con determinaciones analíticas en extractos de suelo.

\section{Literatura citada}

Alling, V.; Hale, S.E.; Martinsen, V.; Mulder, J.; Smebye, A.; Breedveld, G.D. and Cornelissen, G. 2014. The role of biochar in retaining nutrients in amended tropical soils. Journal of Plant Nutrition and Soil Science, 177(5):671-680.

Alloway, B.J. (Comp.). 2013. Heavy metals in soils. 3ra Edición. Springer Science+Business Media, Dordrecht, Países Bajos. 849p.

Amacher, M.C.; Henderson, R.E.; Breithaupt, M.D.; Seale,
C.L. and LaBauve, J.M. 1990. Unbuffered and buffered salt methods for exchangeable cations and effective cation-exchange capacity. Soil Science Society of America Journal, 54(4):1036-1042.

Arenas-Lago, D.; Andrade, M.L.; Lago-Vila, M.; Rodríguez-Seijo, A. and Vega, F.A. 2014. Sequential extraction of heavy metals in soils from a copper mine: Distribution in geochemical fractions. Geoderma, 230231:108-118.

Asensio, V.; Vega, F.A.; Andrade, M.L. and Covelo, E.F. 2013. Technosols made of wastes to improve physico-chemical characteristics of a copper mine soil. Pedosphere, 23(1):1-9.

Bazán T., R. 1996. Manual para el análisis químico de suelos, aguas y plantas. Universidad Nacional Agraria La Molina - Fundación Perú, Lima, Perú. 55 p.

Behera, S.K. and Shukla, A.K. 2015. Spatial distribution of surface soil acidity, electrical conductivity, soil organic carbon content and exchangeable potassium, calcium and magnesium in some cropped acid soils of India. Land Degradation \& Development. 26(1):71-79.

Deressa, A.; Bote, B and; Legesse, H. 2013. Evaluation of soil cations in agricultural soils of east Wollega zone in south western Ethiopia. Science, Technology and Arts Research Journal, 2(1):10-17.

Dolar, S.G. and Keeney, D.R. 1971. Availability of Cu, Zn and $\mathrm{Mn}$ in soils: II. Chemical extractability. Journal of the Science of Food and Agriculture, 22(6):279-282.

Garrels, R.M. and Christ, C.L. 1965. Solutions, minerals, and equilibria. Freeman, San Francisco, California, EEUU. 450 p.

Harris, D.C. 2016. Quantitative chemical analysis. 9na Edición. W.H. Freeman \& Company, New York, 


\section{EEUU. 792 p.}

Hengl, T.; Heuvelink, G.B.M.; Kempen, B.; Leenaars, J.G.B.; Walsh, M.G.; Shepherd, K.D.; Sila, A.; MacMillan, R.A.; Mendes de Jesus, J.; Tamene, L. and Tondoh, J.E. 2015. Mapping soil properties of Africa at 250 m resolution: Random forests significantly improve current predictions. PLoS ONE, 10(6): 0125814. doi:10.1371/journal.pone.0125814.

James, J.; Littke, K.; Bonassi, T. and Harrison, R. 2016. Exchangeable cations in deep forest soils: Separating climate and chemical controls on spatial and vertical distribution and cycling. Geoderma, 279:109-121.

Kim, C.; Choo, G.C.; Cho, H.S. and Lim, J.T. 2015. Soil properties of cultivation sites for mountain-cultivated ginseng at local level. Journal of Ginseng Research, 39(1):76-80.

Lindsay, W.L. 1979. Chemical equilibria in soils. John Wiley \& Sons, New York, EEUU.

Lopes, A.S. and Cox, F.R. 1977. A survey of the fertility status of surface soils under "cerrado" vegetation in Brazil. Soil Science Society of America Journal, 41(4):742-747.

Lu, X.; Mao, Q.; Gilliam, F.S.; Luo, Y. and Mo, J. 2014. Nitrogen deposition contributes to soil acidification in tropical ecosystems. Global Change Biology, 20(12):3790-3801.

Mengel, K. and Kirkby, E.A. 2001. Principles of plant nutrition. 5ta Edición. Kluwer Academic Publishers, Dordrecht, Países Bajos. 849p.

Ott, R.L. and Longnecker, M.T. 2016. An introduction to statistical methods and data analysis. 7ma Edición. Cengage Learning, Boston, Massachusetts, EEUU. $1174 \mathrm{p}$.

Pérez-Esteban, J.; Escolástico, C.; Moliner, A.; Masaguer, A. and Ruiz-Fernández, J. 2014a. Phytostabilization of metals in mine soils using Brassica juncea in combination with organic amendments. Plant and Soil, 377(1-2):97-109.

Pérez-Esteban, J.; Escolástico, C.; Masaguer, A.; Vargas, C. and Moliner, A. 2014b. Soluble organic carbon and $\mathrm{pH}$ of organic amendments affect metal mobility and chemical speciation in mine soils. Chemosphere, 103:164-171.

Ross, D.S.; David, M.B.; Lawrence, G.B. and Bartlett, R.J. 1996. Exchangeable hydrogen explains the $\mathrm{pH}$ of Spodosol Oa horizons. Soil Science Society of America Journal, 60(6):1926-1932.

Shi, L.; Zhang, H.; Liu, T.; Zhang, W.; Shao, Y.; Ha, D.; Li, Y.; Zhang, C.; Cai, X.; Rao, X.; Lin, Y.; Zhou, L.; Zhao, P.; Ye, Q.; Zou, X. and Fu, S. 2016. Consistent effects of canopy vs. understory nitrogen addition on the soil exchangeable cations and microbial community in two contrasting forests. Science of the Total Environment, 553:349-357.

Shuman, L.M. and Duncan, R.R. 1990. Soil exchangeable cations and aluminum measured by ammonium chloride, potassium chloride, and ammonium acetate. Communications in Soil Science and Plant Analysis, 21(13-16):1217-1228.

Sims, J.T. 1996. Lime requirement. En: Sparks, D.L. (Comp.). Methods of soil analysis. Part 3. Chemical methods. 3ra Edición. Soil Science Society of America Book Series No. 5. Soil Science Society of America, Madison, Wisconsin, EEUU. 491-515p.

Stevens, C.J.; Dise, N.B. and Gowing, D.J. 2009. Regional trends in soil acidification and exchangeable metal concentrations in relation to acid deposition rates. Environmental Pollution, 157(1):313-319.

Thomas, G.W. 1996. Soil pH and soil acidity. En: Sparks, D.L. (Comp.). Methods of soil analysis. Part 3. Chemical methods. 3ra Edición. Soil Science Society of America Book Series No. 5. Soil Science Society of America, Madison, Wisconsin, EEUU. 475-490p.

Tomasic, M.; Zgorelec, Z.; Jurisic, A. and Kisic, I. 2013. Cation exchange capacity of dominant soil types in the Republic of Croatia. Journal of Central European Agriculture, 14(3): 84-98.

van der Heijden, G.; Legout, A.; Pollier, B.; Mareschal, L.; Turpault, M.-P.; Ranger, J.and Dambrine, E. 2013. Assessing $\mathrm{Mg}$ and $\mathrm{Ca}$ depletion from broadleaf forest soils and potential causes. A case study in the Morvan Mountains. Forest Ecology and Management, 293:6578.

Yitbarek, T.; Gebrekidan, H.; Kibret, K. and Beyene, S. 2013. Impacts of land use on selected physicochemical properties of soils of Abobo area, western Ethiopia. Agriculture, Forestry and Fisheries, 2(5):177-183. 